\title{
2019 Awards in the Journal of Plant Research
}

\author{
Kouki Hikosaka ${ }^{1}$
}

Published online: 25 June 2019

(c) The Botanical Society of Japan and Springer Japan KK, part of Springer Nature 2019

The Botanical Society of Japan honors excellence in publications of the Journal of Plant Research through the Best Paper Awards and the Most-Cited Paper Award every year. We are proud to announce this year's recipients.

\section{Best paper awards}

Kanako Bessho-Uehara, Jovano Erris Nugroho, Hirono Kondo, Rosalyn B. Angeles-Shim and Motoyuki Ashikari at the Bioscience and Biotechnology Center, Nagoya University, studied development and growth of rhizomes in Oryza longistaminata, the African rice (Bessho-Uehara et al. 2018). Rhizomes of this species elongate horizontally underground as sink organs, but they often undergo a developmental transition that shifts their growth to the surface of the ground to become aerial stems. Although such processes are indispensable for vegetative propagation in clonal plants, their mechanisms have been unclear. The authors found that sucrose specifically represses the developmental transition from rhizome to aerial stem by inhibiting the expression of sugar metabolism and hormone synthesis genes at the bending point. Sucrose depletion affected several factors contributing to the developmental transition of rhizomes including signal transduction, transcriptional regulation, and plant hormone balance. They concluded that sucrose is one of the important factors regulating the developmental transition of rhizomes in $O$. longistaminata. Their findings will contribute to understanding and artificial manipulation of clonal growth.

Atsushi Kume at the Faculty of Agriculture, Kyushu University, and Tomoko Akitsu and Kenlo Nishida Nasahara at the Faculty of Life and Environmental Sciences, University of Tsukuba, discussed the ecological significance of

Kouki Hikosaka

hikosaka@tohoku.ac.jp

1 Graduate School of Life Sciences, Tohoku University, Aoba, Sendai 980-8578, Japan chlorophyll $b$ in light-harvesting systems of photosystems in terrestrial plants (Kume et al. 2018). Terrestrial plants possess two types of chlorophyll, chlorophyll $a$ and $b$. They form light-harvesting chlorophyll-binding protein complexes and their peak wavelength of the absorption spectra differs ca. $20 \mathrm{~nm}$ from each other. The authors addressed the question of why chlorophyll $b$ is absent in the core complexes of photosystems but present only in light-harvesting complexes. Their work indicated that the direct and diffuse solar radiation have different spectral distributions from each other. They discussed the fact that the absorption spectra of chlorophyll $b$ are suitable for absorbing diffuse light but not for avoiding strong direct light. They concluded that the spectra of the photosynthetic pigments and constructed photosystems and antenna proteins significantly align with the terrestrial solar spectra to allow the safe and efficient use of solar radiation. It is worthy of note that Atsushi Kume has published excellent papers about this topic in the Journal of Plant Research (Kume 2017; Kume et al. 2016).

\section{Most-cited paper award}

The article by Wataru Yamori at the Graduate School of Science, The University of Tokyo, is one of the review articles collected for the JPR symposium entitled "Responses of the photosynthetic systems to spatio-temporal variations in light environments: scaling and eco-devo approaches" (Yamori 2016). This article discussed the photosynthetic response to fluctuating environments and photoprotective strategies under abiotic stress. Plants in the natural environment are exposed to diverse, highly dynamic, and unpredictable conditions. To cope with them, plants have various mechanisms that protect the photosynthetic machinery under excess light. This article described the magnitude of environmental fluctuations under natural conditions and the effects of fluctuations in light intensity, $\mathrm{CO}_{2}$ concentration, leaf temperature, and relative humidity on dynamic photosynthesis. Yamori summarized photoprotective strategies that allow plants to maintain photosynthesis under stressful fluctuating environments. 
Kouki Hikosaka

Editor-in-Chief, Journal of Plant Research

\section{References}

Bessho-Uehara K, Nugroho JE, Kondo H, Angeles-Shim RB, Ashikari M (2018) Sucrose affects the developmental transition of rhizomes in Oryza longistaminata. J Plant Res 131:693-707

Kume A (2017) Importance of the green color, absorption gradient, and spectral absorption of chloroplasts for the radiative energy balance of leaves. J Plant Res 130:501-514
Kume A, Akitsu T, Nasahara KN (2016) Leaf color is fine-tuned on the solar spectra to avoid strand direct solar radiation. J Plant Res 129:615-624

Kume A, Akitsu T, Nasahara KN (2018) Why is chlorophyll $b$ only used in light-harvesting systems? J Plant Res 131:961-972

Yamori W (2016) Photosynthetic response to fluctuating environments and photoprotective strategies under abiotic stress. J Plant Res 129:379-395

Publisher's Note Springer Nature remains neutral with regard to jurisdictional claims in published maps and institutional affiliations. 